\title{
Infrared Image Segmentation Algorithm Using Histogram-Based Self-adaptive K-means Clustering
}

\author{
Zhiqiang Zhao ${ }^{1, \mathrm{a}}$, Xin Ling ${ }^{2, \mathrm{~b}}$, Jian $\mathrm{Wu}^{3}$, XiaoyongRui ${ }^{4}$ \\ ${ }^{1,3,4}$ School of Bioinformatics, Chongqing University of Posts and Telecommunications, Chongqing, \\ China \\ ${ }^{2}$ Academy of Automation, Chongqing University of Posts and Telecommunications, Chongqing, \\ China \\ a3927545@qq.com, bsy8098496@163.com
}

Keyword: K-means, Histogram, Infrared Image Segmentation, Human Detection

\begin{abstract}
For the problem that the different parameters of infrared imaging equipment and the environment around the target cause the poor robustness of threshold value automatic acquisition method in infrared human target segmentation algorithm, starting from the principle of infrared imagery and connecting with the characteristics of the histogram and K-means clustering algorithm, we propose an infrared image segmentation algorithm using histogram-based self-adaptive K-means clustering. We use histogram peaks to determine the $\mathrm{K}$ ' value of $\mathrm{K}$-means clustering and select the grey values corresponding to this $\mathrm{K}$ peaks as the $\mathrm{K}$ initial cluster center values of clustering algorithm. After clustering, we select appropriate trough as a segmentation point through the cluster center's moving direction. This algorithm does not require to balance the image beforehand and to suppose background distribution. The experimental results show that the algorithm is simple and flexible, easy to implement, and has good robustness.
\end{abstract}

\section{Introduction}

Compared with visible imaging, infrared imaging, especially the far-infrared passive imaging technology, has a unique advantage.Infrared imaging is the object of the thermal imaging, with strong ability of "penetration". Infrared imaging is hardly affected by light conditions and environment, possessing the ability to penetrate the darkness and smoke for all-weather work.Consequently, human detection technology in infrared image is widely used in various fields ${ }^{[1-3]}$, such as close military reconnaissance and strike, security monitoring (intruder detection) ofborder and special places, personnel rescue, auxiliary driving (pedestrian detection), man-machine interface, robot vision, etc.

The core technology of the human detection in infrared image is fast segmenting and classifying the human body target in the scene. The accurate segmentation of the target is the basis and key of the whole detection system ${ }^{[4]}$. Butthe different parameters of infrared imaging equipment and the environment around the target cause the poor robustness of threshold value automatic acquisition method in infrared human target segmentation algorithm. To improve the robustness of threshold value selection in infrared human target segmentation algorithm, we propose an infrared image segmentation algorithm using histogram-based self-adaptive K-means clustering.

\section{Analysis of Infrared Image Segmentation Algorithm}

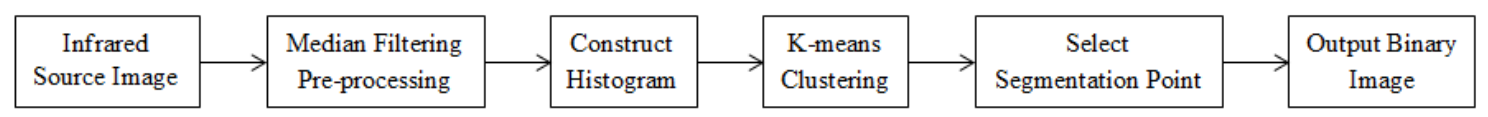

Fig. 1

\subsection{Median Filtering Pre-processing of Infrared Source Image}

Median filter is a classical method to smooth noise. It can well filter impulse noise and salt and pepper noise and well protect the edge of the signal from being blurred. Its basic principle is 
substituting the mid-value of all points in the field of a point for this point to eliminate the isolated noise points ${ }^{[5]}$.

2D median filter can be defined as

$$
g(x, y)=\operatorname{med}\{f(x-i, y-j),(i, j \in W)\}
$$

Among this formula, $f(x, y)$ is the original image and $g(x, y)$ is the processed image. $W$ is 2Dtemplate, and usually it is a rectangular region such as $3 * 3$ or $5 * 5$. Also, it may be line, round, cross or annulus.

The concrete method is as follow. Use a structure of 2D slide template to rank the points in the field of thepoint to be processed according to their pixels. Then produce a 2Ddata sequence which increases or decreases monotonically, and substitute the mid-value of the sequence for the point to be processed.

\subsection{Gray Histogram Statistics}

Traditional K-means clustering needs to artificially set the initial cluster number, namely, the initial value of $\mathrm{K}$. For an infrared image, if the contrast between the foreground target and the background region is big, it is easy to determine the initial value of $\mathrm{K}$; however, if the contrast between the foreground target and the background region is not big, it is hard to determine the initial value of $\mathrm{K}^{[6-7]}$.

Histogram is the most basic statistical characteristic of image. It shows the number of every kind of grey level in an image, and reflects the frequency of every kind of grey level in an image ${ }^{[8-9]}$. The peaks of histogram show this grey level appear frequently in the image, and the targets composed of the pixels near the peaks usually belong to the same category. So, we can believe that the peaks of histogram are equal to the categories of the targets in the image. In other words, we can use the peaks to determine the cluster number $\mathrm{K}$. In addition, the valleys of histogram show this grey level few appear in the image, and they are usually between different categories of targets. So, we can select a valley as the segmentation point.

\subsection{Histogram-Based Self-adaptive K-means Clustering}

We need not artificially set the initial value of $\mathrm{K}$ and can make it to cluster self-adaptively if using the number of the peaks as $\mathrm{K}$. And we can use the grey levels of the peaks as the $\mathrm{K}$ initial central values of clustering algorithm to avoid the randomness of selecting the initial cluster centers in traditional K-means clustering.

The algorithm flow is as follow.

Step 1: Use the number of the peaks in the histogram as the cluster number $\mathrm{K}$, and use the grey levels of the peaks as the $\mathrm{K}$ initial central values of clustering algorithm.

Step 2: Calculate the Euclidean distances from every point to the K cluster centers, then put every point in the categories that their nearest center belong to.

Step 3: Calculate the new central values of every cluster and the variable quantity between old and new central values.

Step 4: If the variable quantity between the old and new central values is less than the given threshold value, the clustering is complete. Otherwise, repeat Step 2 and Step 3.

\subsection{Segmentation Threshold Value Selection}

2.4.1 When there is only one peak in the histogram, namely, $K=1$, we select the valley close to the maximal gray level as the segmentation point because of that the less bright background region usually accounts for a higher proportion of the infrared image while the more bright foreground region accounts for a lower proportion. For instance, Table 1 and Fig. 2 show that there are one peak $u_{1}$ and two valleys $v_{1}$ and $v_{2}$ in the histogram and the valley $v_{2}$ close to the maximal gray level can be the segmentation point. 
Table 1

\begin{tabular}{|c|c|}
\hline \multicolumn{2}{|c|}{1} \\
\hline \multicolumn{2}{|c|}{83} \\
\hline 1 & 2 \\
\hline 53 & 116 \\
\hline
\end{tabular}

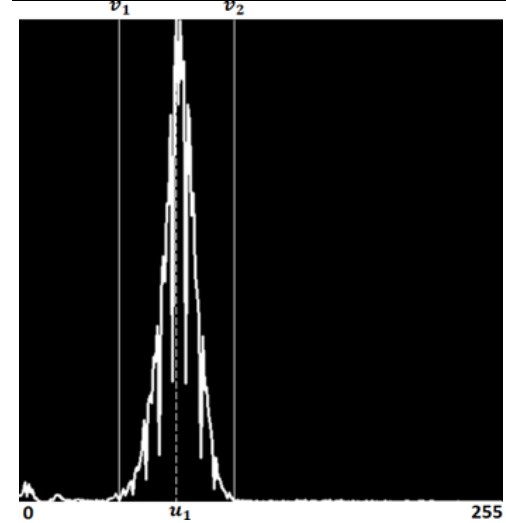

Fig. 2

2.4.2When there is more than one peak in the histogram, namely, $\mathrm{K} \geq 2$, the valleys can be classified into three types, the valleys between the two peaks, the valleys between the peaks and the maximal gray level and the valleys between the peaks and the minimal gray level.

The variable quantity $\Delta u_{i}$ between the initial center (the peak) $u_{i}$ and the center $u_{i}{ }^{\prime}$ when the clustering is complete reveal the moving direction of cluster center.

A. For the valleys between the two peaks, the position changes of these two peaks can be used to determine whether the valleys can be the segmentation point.

a. If these two cluster centers move face-to-face and get close to each other, it means that there are few pixels between these two clusters. This type of valleys is the border of two different categories of targets and can be the segmentation point. For instance, Table 2 and Fig. 3 show that the valley $v_{2}$ between $u_{2}$ and $u_{3}$ can be the segmentation point.

b. If these two cluster centers move back-to-back and get away from each other, it means that there are a large number of pixels which belong to the same category of target between these two clusters. So, this type of valleys cannot be the segmentation point. For instance, Table 3 and Fig. 4 show that the valley $v_{2}$ between $u_{1}$ and $u_{2}$ cannot be the segmentation point.

c. If these two cluster centers move in the same direction, it means that there is a region which has few pixels in their moving direction while there are a large number of pixels which belong to the same category in the opposite direction. So, this type of valleys cannot be the segmentation point. For instance, Table 2 and Fig. 3 show that the valley $v_{1}$ between $u_{1}$ and $u_{2}$ cannot be the segmentation point and Table 4 and Fig. 5 show that the valley $v_{1}$ between $u_{1}$ and $u_{2}$ cannot be the segmentation point, too.

B. For the valleys between the peaks and the maximal gray level, if the cluster center moves in the positive direction, namely, close to the maximal gray level and there is no other valley, this type of valleys can be the segmentation point. For instance, Table 3 and Fig. 4 show that the valley $v_{2}$ between $u_{2}$ and the maximal gray level can be the segmentation point and Table 4 and Fig. 5 show that the valley $v_{2}$ between $u_{2}$ and the maximal gray level can be the segmentation point, too.

C. For the valleys between the peaks and the minimal gray level, the less bright background region usually accounts for a higher proportion of the infrared image while the more bright foreground region accounts for a lower proportion, so this type of valleys cannot be the segmentation point. For instance, Table 3 and Fig. 4 show that the valley $v_{1}$ between $u_{1}$ and the minimal gray level cannot be the segmentation point. 
After determining the segmentation point by using the above method, the grey value of this point is used as the threshold value in the image binary progress and the segmentation result is obtained.

Table 2

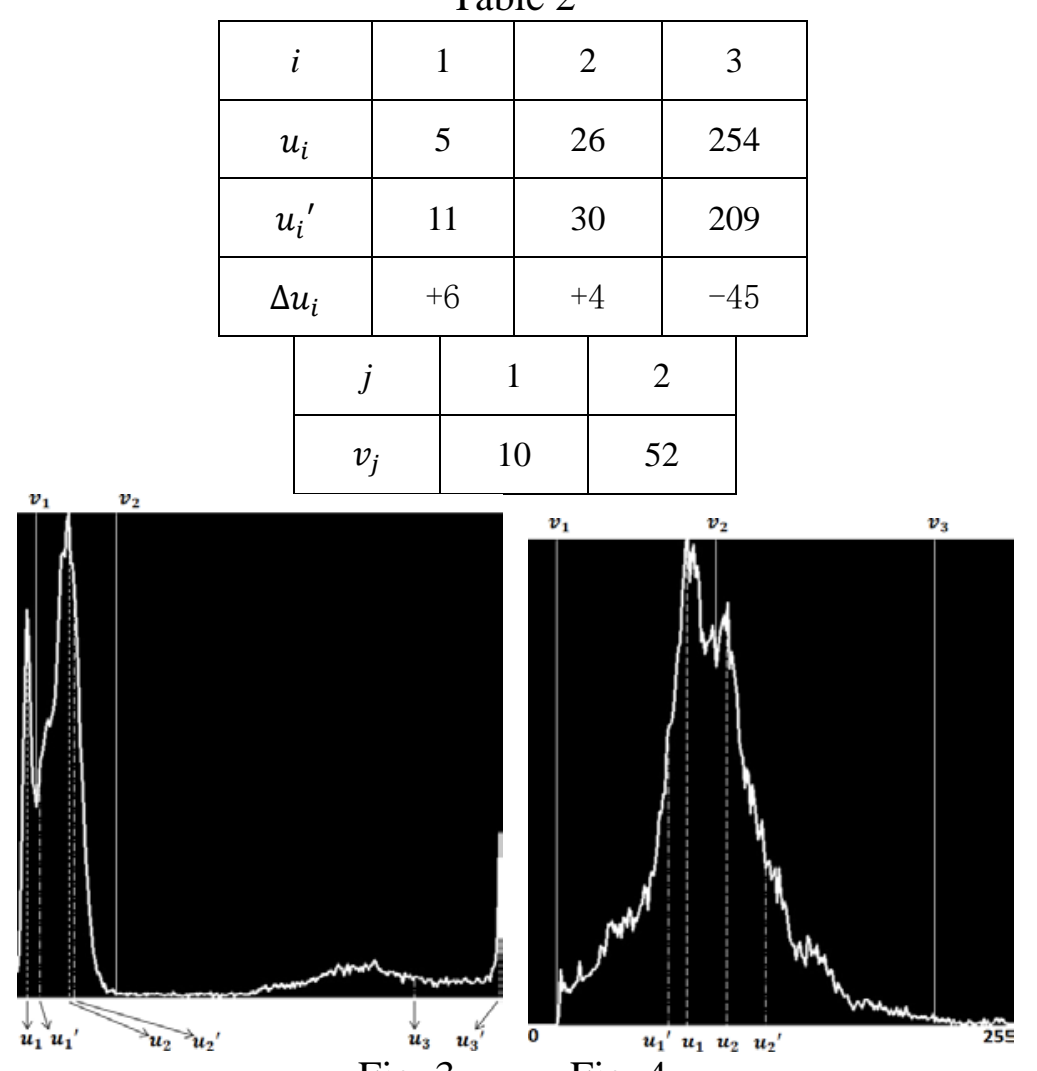

Fig. 3

Fig. 4

Table 3

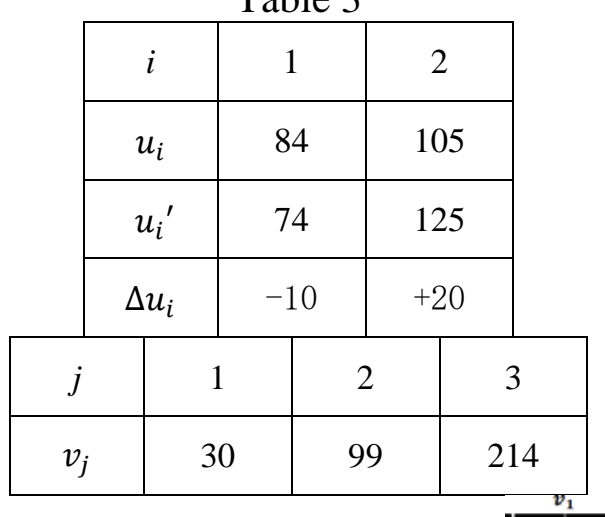

Table 4

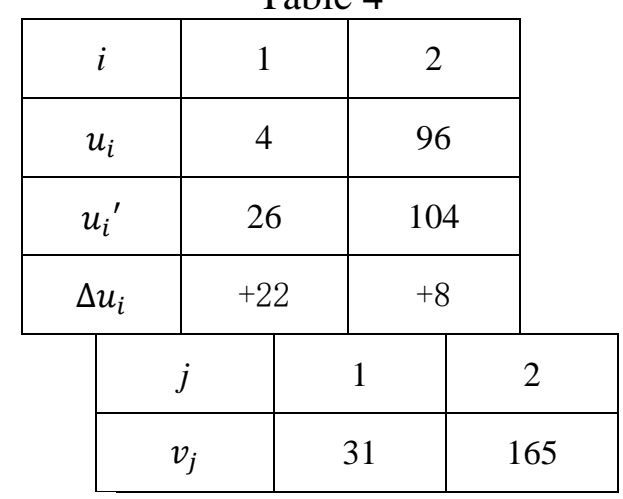

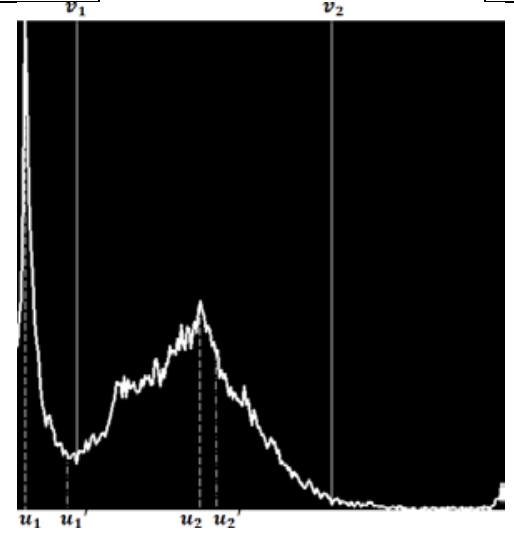

Fig. 5 


\section{Experimental Results and Analysis}

We obtain relatively satisfactory results by using the proposed algorithm to segment several hundred infrared images which using different equipment shooting in different time and different places and more than ten infrared image sequences such as OSU Thermal Pedestrian Database, OSU Color-Thermal Database, Terravic Weapon Infrared Database and so on. In addition, we compare the proposed algorithm with other algorithms (K-means clustering algorithm $(K=2)$, Otsu algorithm ${ }^{10]}$ and P-tile threshold value segmentation algorithm ${ }^{[11]}(\mathrm{P}=0.2)$ are used.) and the experimental result is as follow. The segmentation quality of K-means clustering is good when the contrast between the foreground target and the background region is big while the segmentation quality is bad when the contrast is not big. In most situations, the segmentation quality of Otsu is unsatisfactory because the distribution of the histogram of the infrared image is not the ideal bimodality and theinfrared image cannot be divided into the foreground and background by maximum between-cluster variance method.The segmentation quality of P-tilethreshold value segmentation is good when the actual size of the target is nearly equal to the size of the previous estimates. But in most situations, the segmentation quality is bad because the proportion of the target is unknown.

In sum, the proposed algorithm has good robustness and self-adaptability, clear and intact segmentation result and good segmentation quality.

(a: Infrared source image, b: K-means clustering algorithm, c: Otsu algorithm, d: P-tile threshold value segmentation algorithm, e: the proposed algorithm)

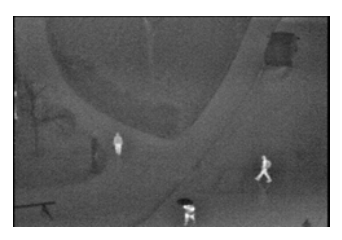

Fig. 6.1(a)

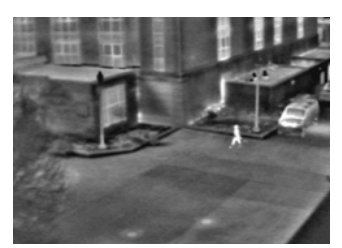

Fig. 6.2(a)

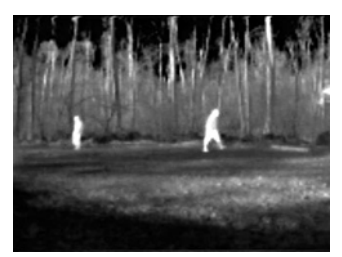

Fig. 6.3(a)

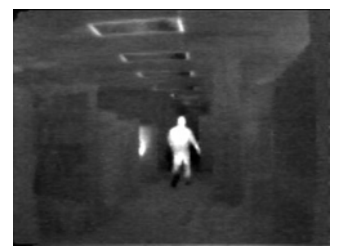

Fig. 6.4(a)

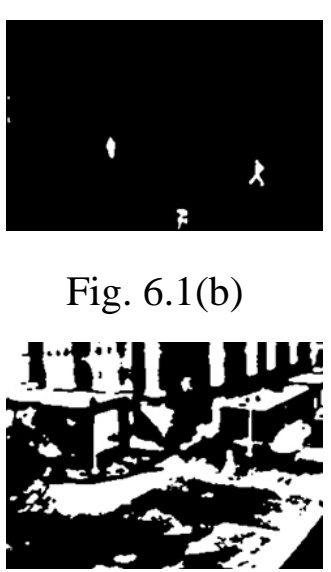

Fig. 6.2(b)

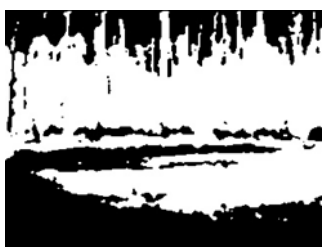

Fig. 6.3(b)

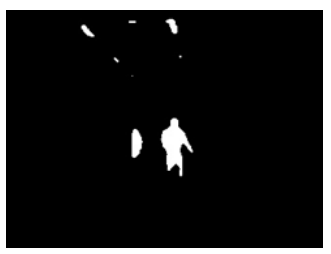

Fig. 6.4(b)

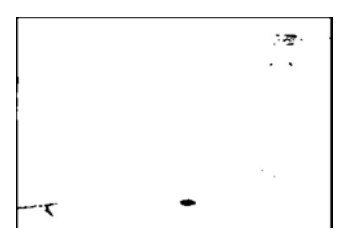

Fig. 6.1(c)

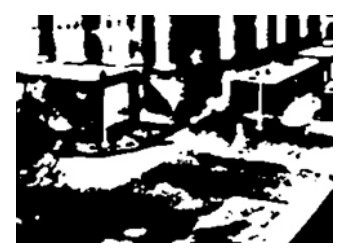

Fig. 6.2(c)

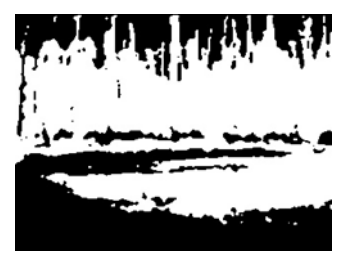

Fig. 6.3(c)

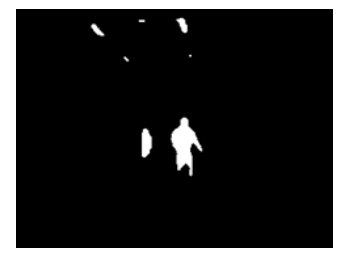

Fig. 6.4(c)

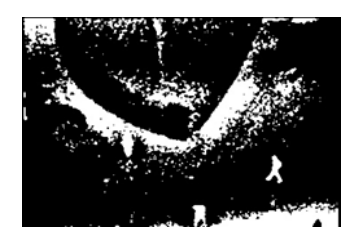

Fig. 6.1(d)

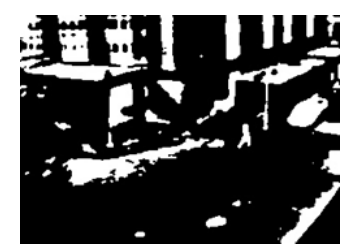

Fig. 6.2(d)

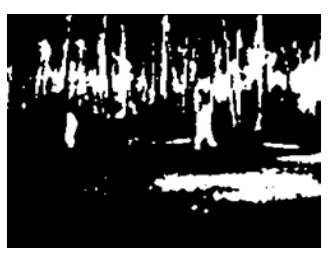

Fig. 6.3(d)

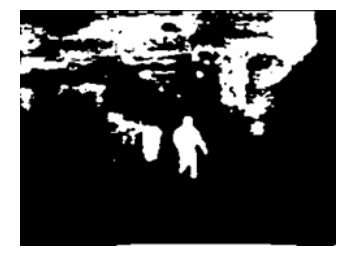

Fig. 6.4(d)

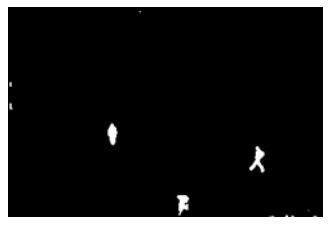

Fig. 6.1(e)

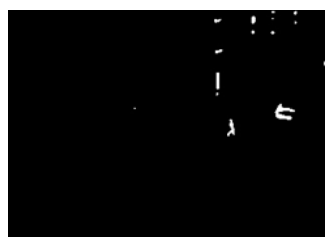

Fig. 6.2(e)

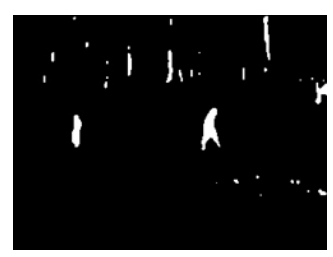

Fig. 6.3(e)

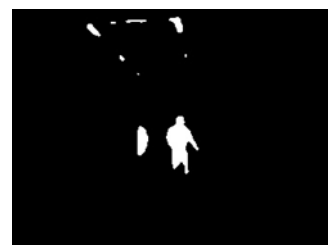

Fig. 6.4(e) 


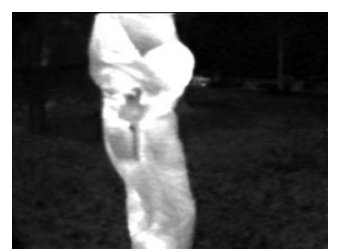

Fig. 6.5(a)

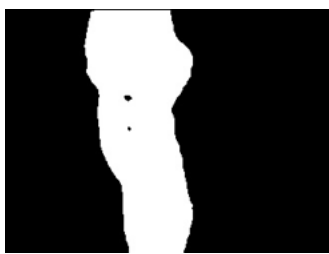

Fig. 6.5(b)

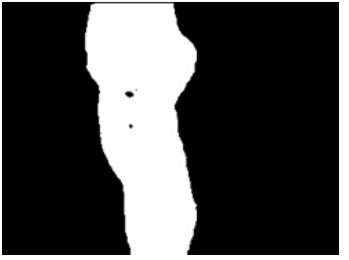

Fig. 6.5(c)

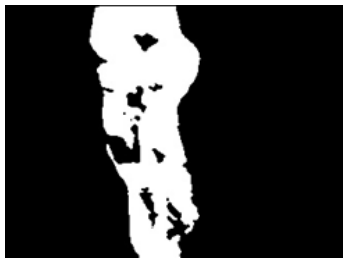

Fig. 6.5(d)

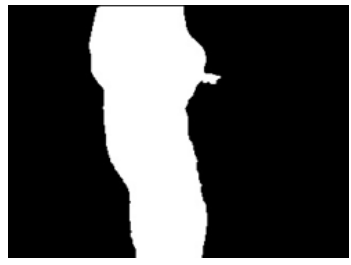

Fig. 6.5(e)

\section{Summary}

In this paper, starting from the principle of infrared imagery and connecting with the characteristics of the histogram and K-means clustering algorithm, we propose an infrared image segmentation algorithm using histogram-based self-adaptive K-means clustering. We need not artificially set the initial value of $\mathrm{K}$ and can make it to cluster self-adaptively by using the number of the peaks as K. This method can be used to determine the cluster number quickly. We can use the grey levels of the peaks as the $\mathrm{K}$ initial central values of clustering algorithm to avoid the randomness of selecting the initial cluster centers in traditional K-means clustering. This method reduces the calculation capacity and increases the calculation efficiency. We select the appropriate valley as the segmentation point by the moving direction of the cluster centers. This method improves the robustness of infrared image segmentation. The proposed algorithm, obtaining the good binary-conversion effect, reduces the number of the non-objected regions and the complexity of the subsequent image analysis. In the QT IDE on the Linux OS (CPU: 2.6GHz RAM: 4GB), the proposed algorithmuses $0.01 \mathrm{~s}$ to segment an infrared image, with the help of the OpenCV. It can completely meet the real-time requirements.

\section{References}

[1]. Tarak Gandhi, Mohan ManubhaiTrivedi. Pedestrian Protection Systems: Issues, Survey and Challenges [J]. IEEE TRANSACTIONS ON INTELLIGENT TRANSPORTATION SYSTEMS, 2007, 8(3): 413-430.

[2]. Ronan O’Malley, Martin Glavin, Edward Jones. A review of automotive infrared pedestrian detection techniques [C]. IET Signals and Systems Conference, Galway, Irish, 2008: 168-173.

[3]. A.Shahua, Y.Gdlyhu and G.Hayun. Pedestrian detection for driving assistance system: single-frame classification and system level performance [C]. Proceedings of IEEE Intelligent Vehicles Symposium, 2004: 1-6.

[4]. M Bertozzi, ABroggi, P Grislerietal. Pedestrian Detection in Infrared Images [C]. Proceedings of the IEEE International Conference on Intelligent Transportation Systems, Shanghai, 2003, 662667.

[5]. Wei Sun, Liangzheng Xia. Infrared target segmentation algorithm based on morphological method [J]. Infrared Millim. Waves, 2004, 23(3): 232-236. (in Chinese with an English abstract)

[6]. A. Neri, S. Colonnese, G. Russo, P. Talone. Automatic moving object and background separation [J]. Signal Processing, 1998, 66(2): 219-232.

[7]. JiaGuo, Wengang Qin, Weiguo Liu. Adaptive algorithm for infrared target enhancement [J]. Journal of Applied Optics, 2009, 30(2): 357-360. (in Chinese with an English abstract)

[8]. A. El Maadi, X. Maldague. Outdoor infrared video surveillance: a novel dynamic technique for the subtraction of a changing background of IR images [J]. Infrared Physics \& Technology, 2007, 49(3): 261-265. 
[9]. J. Han, B. Bhanu. Human activity recognition in thermal infrared imagery [C]. Proceedings of IEEE Computer Society Conference on Computer Vision and Pattern Recognition, 2005.

[10]. Otsu N. A threshold selection method from gray-level histogram [J]. IEEE Transactions on Systems, Man, and Cybernetics, 1979, 9(1): 62-66.

[11]. Yasuno M, Ryousuke S, Yasuda N, et al. Pedestrian detection and tracking in far infrared images[C]// Proceedings of IEEE Intelligent Transportation Systems. Vienna, Austria: IEEE, 2005: 182-187. 